\title{
EchoGéo
}

25 | 2013

Moyen-Orient : espaces et passeurs du changement

\section{Une lecture spatiale des politiques des États du Proche-Orient}

Compte rendu de lecture

Roman-Oliver Foy et Jack Keilo

\section{OpenEdition}

\section{Journals}

Édition électronique

URL : https://journals.openedition.org/echogeo/13508

DOI : 10.4000/echogeo.13508

ISSN : 1963-1197

Éditeur

Pôle de recherche pour l'organisation et la diffusion de l'information géographique (CNRS UMR 8586)

Référence électronique

Roman-Oliver Foy et Jack Keilo, « Une lecture spatiale des politiques des États du Proche-Orient », EchoGéo [En ligne], 25 | 2013, mis en ligne le 10 octobre 2013, consulté le 10 août 2021. URL : http:// journals.openedition.org/echogeo/13508 ; DOI : https://doi.org/10.4000/echogeo.13508

Ce document a été généré automatiquement le 10 août 2021.

EchoGéo est mis à disposition selon les termes de la licence Creative Commons Attribution - Pas d'Utilisation Commerciale - Pas de Modification 4.0 International (CC BY-NC-ND) 


\title{
Une lecture spatiale des politiques des États du Proche-Orient
}

\author{
Compte rendu de lecture
}

Roman-Oliver Foy et Jack Keilo

1 Référence de l'ouvrage : Atlas du Proche-Orient arabe, de Fabrice Balanche. Paris : PUPS, 2012, 140 p., 300 cartes et graphiques, ISBN : 978-2-84050-797-0

2 L'Atlas du Proche-Orient arabe est le premier du genre en français. Jusqu'à aujourd'hui, les atlas sur la région à cette échelle étaient essentiellement historiques (Hours, Auranche, Cauvin, et al., 1994 ; Roaf, 1991 ; Schyle, 1992). D’autres avaient été réalisés à des échelles plus petites à propos de géopolitique (CIA, 1993; Lemarchand, 1993; Vallaud, Baron, 2010), ou plus grandes (Gilbert, 2008 ; Lemarchand, Radi, 1996 ; Verdeil, Faour, Velud, 2007 ; Roujon, Vilan, 2010). L'ouvrage de Fabrice Balanche permet donc de combler un manque en traitant spécifiquement du « Proche-Orient arabe " à travers des problématiques aussi bien politiques et culturelles qu'économiques et sociales.

Les trois premiers chapitres permettent de présenter les caractéristiques physiques de la région et d'en dresser l'histoire récente. Une dialectique apparaît constamment entre la formation des communautés et leur instrumentalisation par les États impérialistes, avant les indépendances, puis nationaux à partir de la seconde moitié du vingtième siècle. Dans le premier chapitre, l'auteur trouve dans le climat et le relief des facteurs explicatifs de la répartition de la population en insistant sur les oppositions culturelles : minorités confessionnelles dans les montagnes face aux «religions du pouvoir » en plaine. Il pointe aussi les risques actuels de surexploitation des ressources, notamment l'eau et les sols, à cause de la croissance urbaine et de l'extension des superficies irriguées. Le second chapitre vise à démontrer le rôle des métropoles ottomane et occidentales dans le découpage politique actuel de la région et les clivages entre communautés d'une part et entre nomades et sédentaires d'autre part. Le troisième chapitre présente l'émergence des États comme forces de modernisation et de dépassement des contrastes communautaires grâce au nationalisme arabe. Trois structures différentes émergent de ces constructions étatiques : la Syrie est centralisée 
autour de Damas ; le Liban est éclaté entre des communautés antagonistes ; la majorité de la population jordanienne vient de Palestine et la monarchie hachémite d'Arabie.

Les trois chapitres suivants traitent des dynamiques socioéconomiques de la région où la croissance démographique pèse sur des économies qui stagnent. Le quatrième chapitre montre que le Proche-Orient arabe a connu sa transition démographique dans la deuxième moitié du vingtième siècle. Mais la baisse récente de la natalité, grâce à l'éducation et à l'essor du travail féminin, suggère que la région entre dans un régime post-transitionnel. Ces dynamiques sont ensuite analysées sous le prisme d'une lecture communautaire des sociétés proche-orientales à travers la description des différentes vagues migratoires et des disparités dans le poids démographique de chaque confession. Le cinquième chapitre cherche à expliquer l'évolution des structures économiques au Proche-Orient en détaillant les stratégies politiques de nationalisation et de libéralisation menées par les États et les enjeux de géopolitique régionale. Le développement économique serait limité par le manque de ressources (agricoles et énergétiques par exemple), la corruption de l'appareil bureaucratique en Syrie et les tensions politiques au Liban. En ce qui concerne les flux de marchandises et d'énergie, l'ouvrage démontre la marginalisation que subit la façade levantine. Le contournement du Proche-Orient est le résultat des fragilités politiques et économiques des pays de la région et reflètent le déplacement du centre de gravité économique vers le Golfe. Le sixième chapitre montre comment la croissance démographique a essentiellement été absorbée par les grandes métropoles. F. Ballanche souligne les caractéristiques de la croissance de chacune d'entre elles : l'étalement urbain de Damas se fait au détriment de la qualité environnementale; Amman est construite à l'image des villes du Golfe ; Beyrouth est une ville marquée par les clivages confessionnels et sociauxéconomiques; et Alep, après avoir connu un déclin, profite de l'ouverture économique de la Syrie depuis les années 2000.

5 Le septième chapitre sur les territoires palestiniens semble un peu esseulé dans le propos général de l'ouvrage. L'auteur affirme dès l'introduction sa volonté de traiter cet espace dans un "chapitre particulier» car "son destin lui échappe du fait de l'occupation israélienne ». C'est la raison pour laquelle Israël, bien qu'étant considéré hors du Proche-Orient arabe, est aussi traité "pour comparer ses indicateurs socioéconomiques " à ceux des territoires palestiniens. L'auteur montre que dans cet espace la population augmente rapidement en raison de la compétition démographique entre Palestiniens et Israéliens. Il en résulte une forte croissance urbaine de part et d'autre du mur de séparation. Après avoir montré l'absence d'indépendance économique des territoires palestiniens, notamment à l'égard d'Israël, l'auteur présente les fragilités de l'appareil productif palestinien, essentiellement tertiaire et qui ne pourvoie pas suffisamment d'emplois. Enfin, les enjeux du partage du territoire sont présentés à l'échelle de l'ancienne Palestine mandataire d'une part, et à celle de Jérusalem d'autre part. Le dernier chapitre traite des conflits pour le contrôle de l'espace proche-oriental. L'accent est mis sur les tensions interétatiques pour l'accès aux ressources, notamment hydrauliques, et l'occupation israélienne de certains territoires (notamment le Sud-Liban et le Golan). L'angle adopté pour traiter de ces questions est l'antagonisme entre Israël et les États arabes. L'influence des puissances régionales comme la Turquie, l'Iran, les États-Unis et l'ex-URSS explique les équilibres actuels et passés. 
6 L'ouvrage est bien documenté, avec beaucoup d'informations sur une région où les données sont soit rares et difficilement accessibles, soit si abondantes qu'elles en deviennent inutilisables (par exemple sur le conflit israélo-palestinien). L'auteur a utilisé des sources nombreuses et diversifiées, notamment des ouvrages de référence et des statistiques officielles. Auxquelles s'ajoutent les données qualitatives et quantitatives collectées lors de ses nombreux séjours de terrain et qui dénotent une profonde connaissance de cette région. Cet ouvrage couvre beaucoup de thématiques afin d'offrir au lecteur une image exhaustive de ces pays, notamment à travers le développement économique et social, la diversité culturelle et la géopolitique.

7 Dans ces conditions, traiter de quatre pays différents au sein d'un seul ouvrage de façon synthétique n'était pas facile. Cet atlas parvient à être clair grâce à ses nombreuses figures et à l'efficacité et la simplicité des textes. Les types de cartes sont variés, ce qui permet de multiplier les indicateurs. De même, le passage d'une échelle à l'autre (du quartier au Monde) ouvre les horizons du lecteur et démontre que les phénomènes (par exemple économiques ou culturels) se traduisent de manières différentes en fonction de l'étendue de l'espace observé. Les photographies des paysages ruraux et urbains sont nombreuses et montrent une réalité que les cartes et les chiffres ne peuvent communiquer. Malgré cette diversité, la simplicité des figures permet de ne pas perdre le lecteur dans une description trop complexe de la situation du Proche-Orient arabe.

Néanmoins, ces simplifications ont nécessité des choix qui posent certaines questions. Les confessions sont représentées par des aplats de couleur. Cette façon de cartographier des appartenances religieuses qui sous-entend une occupation exclusive de l'espace par une seule communauté risque de masquer la mixité du peuplement dans la réalité. En outre, le choix de représenter dans une même carte certaines données socioéconomiques avec des procédés de généralisation différents en fonction des divisions administratives ne permet pas de comprendre comment les chiffres ont été obtenus. Par exemple, dans quelques cartes (p. 51-53-54-56-60-69), les régions « semidésertiques » et "désertiques » sont considérées comme non habitées et ne sont à cet égard affectées d'aucun chiffre. Pourtant, ces régions sont comprises dans les divisions administratives des pays à l'échelle desquelles les chiffres ont été calculés. On ne sait pas non plus à partir de quelles données cette zone est considérée comme non habitée alors qu'une grande partie est qualifiée de semi-désertique (p.16), que l'activité principale y est l'élevage nomade (p. 66) et surtout qu'elle comprend une ville, Palmyre, et d'autres villages (p. 50). Enfin, il aurait pu être utile de renvoyer à plus de références pour justifier les données de certaines cartes. Par exemple l'article de F. Balanche: Damas la tentaculaire (2006), dont il n'est pas fait mention dans l'atlas, permettrait de mieux comprendre comment la carte de Damas (p. 84-85) a été réalisée.

Même si de nombreuses thématiques ont été couvertes, certains regretteront que la géographie physique ait si peu de place même si cette absence peut être justifiée par le fait que l'auteur a surtout voulu mettre en avant les dynamiques socioéconomiques, politiques et culturelles. Les questions des droits de l'homme et des capacités militaires sont également absentes. Pourtant de telles informations auraient été utiles pour mieux comprendre par exemple les évènements actuels en Syrie ou la guerre de juillet 2006 au Liban. Quelques pages auraient également pu être consacrées aux représentations cartographiques du «Proche-Orient arabe » à travers le monde. Elles auraient permis de mieux justifier le cadre spatial et de montrer que toute délimitation 
a des implications politiques et peut être utilisée pour construire ou contester une identité.

Pour l'auteur, « le Proche-Orient (...) regroupe les pays concernés par le conflit israéloarabe: Syrie, Liban, Jordanie, Palestine et Israël. L'Égypte a quitté le Proche-Orient lorsqu'elle a signé les accords de Camp David en 1978 ». Pourtant les Palestiniens ont signé les accords d'Oslo en 1993 et les Jordaniens les accords de Wadi Araba en 1994. Dans ces conditions, quelle est la différence entre ces deux pays et l'Égypte? Cette expression, "Proche-Orient arabe », est surtout employée en langue française et ses équivalents arabes et anglais sont moins répandus. N'est-ce pas adopter un point de vue qui implicitement confirme le clivage entre Israël et États arabes alors que des conflits existent également entre Arabes eux-mêmes (par exemple, "Septembre noir ", la guerre civile au Liban ou la crise syrienne actuelle) et que la société israélienne est fragmentée ? Comment ne pas considérer ce clivage comme un prétexte pour justifier les rapports de domination au sein des États de la région et pourquoi dans ces conditions ne pas chercher à le dépasser? Intégrer Israël dans le cadre spatial de l'ouvrage et le traiter comme les autres États (et pas uniquement dans une avantdernière partie consacrée aux territoires palestiniens) n'aurait-il pas mieux mis en perspective les enjeux géopolitiques dans la région? Comparer terme à terme Israël et les autres États de la région, au lieu de toujours laisser le premier de cöté, permettrait une analyse plus complexe et compréhensive des dynamiques régionales au ProcheOrient.

\section{BIBLIOGRAPHIE}

Balanche F., 2006. Damas, la tentaculaire. In Villes et territoires du Moyen-Orient, $\mathrm{n}^{\circ} 2$, http:// www.ifporient.org/sites/default/files/Damas_la_tentaculaire_version_PDF.pdf, consulté le 15 novembre 2012

C.I.A., 1993. Atlas of the Middle East. Washington D.C., The Agency, 76 p.

Gilbert M., 2008. The routledge atlas of the Arab-Israeli conflict. London, Routledge, $205 \mathrm{p}$.

Hours F., Arenche O., Cauvin J., et al., 1994. Atlas des sites du Proche-Orient (14000-5700 BP). Lyon, Maison de l'Orient et de la Méditerranée, 2 vol., 522 p.

Lemarchand P., 1993. Atlas géopolitique du Moyen-Orient et du monde arabe : le croissant des crises. Bruxelles, Ed. Complexe, $284 \mathrm{p}$.

Lemarchand P., Radi L., 1996. Israël-Palestine demain : atlas prospectif. Bruxelles, Ed. Complexe, $143 \mathrm{p}$.

Roaf M., Talon P., 1991. Atlas de la Mésopotamie et du Proche-Orient ancien. Turnhout (Belgique), Brepois, $237 \mathrm{p}$.

Roujon Y., Vilan L., 2010. Les faubourgs de Damas. Atlas contemporain des faubourgs anciens : formes, espaces et perspectives. Damas, Institut Français du Proche-Orient, 396 p. 
Schyle D., 1992. Near Eastern Upper Paleolithic Cultural Stratigraphy : an evaluation of evidence. Wiesbaden, Reichert, $150 \mathrm{p}$.

Vallaud P., Baron X., 2010. Atlas géostratégique du Proche et du Moyen-Orient. Paris, Perrin, 175 p.

Verdeil E., Faour G., Velut S., 2007. Atlas du Liban : territoires et société. Beyrouth, Institut Français du Proche-Orient, 207 p.

\section{AUTEURS}

\section{ROMAN-OLIVER FOY}

Roman-Oliver Foy, foy.roman@gmail.com, est doctorant en géographie, Université Paris 1 Panthéon-Sorbonne, UMR 8185 ENeC, ATER à l'IEDES.

\section{JACK KEILO}

Jack Keilo, keilojack@hotmail.com, est doctorant en géographie, Université Paris 4 Sorbonne, UMR 8185 ENeC, ATER à l'Université de Poitiers. 